Research paper

\title{
Neurochemical differences between bipolar disorder type I and II in superior temporal cortices: A proton magnetic resonance spectroscopy study
}

\author{
Murat İlhan Atagün ${ }^{\mathrm{a}, \mathrm{b}, *}$, Elif Muazzez Şıkoğluc ${ }^{\mathrm{c}}$, Serdar Süleyman Can ${ }^{\mathrm{a}, \mathrm{b}}$, \\ Görkem Karakaş Uğurlu ${ }^{\mathrm{a}, \mathrm{b}}$, Semra Ulusoy Kaymak ${ }^{\mathrm{b}}$, Ali Çayköylüi,b ${ }^{\mathrm{a}, \mathrm{b}}$, Oktay Algın ${ }^{\mathrm{d}, \mathrm{e}}$, \\ Mary L Phillips ${ }^{f}$, Constance M Moore ${ }^{c}$, Dost Öngürrs,h \\ a Department of Psychiatry, Ankara Yuldırtm Beyazt University Medical School, Ankara, Turkey \\ ${ }^{\mathrm{b}}$ Department of Psychiatry, Ankara Atatürk Training and Education Hospital, Ankara, Turkey \\ ${ }^{c}$ Center for Comparative NeuroImaging, Department of Psychiatry and Radiology, University of Massachusetts Medical School, Worcester, MA, USA \\ d Department of Radiology, Ankara Atatürk Training and Education Hospital, Ankara, Turkey \\ e National MR Research Center and Aysel Sabuncu Brain Research Center, Bilkent University, Ankara, Turkey \\ ${ }^{\mathrm{f}}$ Department of Psychiatry, University of Pittsburgh, Medical School, Pittsburgh, PA, USA \\ ${ }^{\mathrm{g}}$ Psychotic Disorders Division, McLean Hospital (Belmont), MA, USA \\ ${ }^{\text {h }}$ Department of Psychiatry, Harvard Medical School, Boston, MA, USA
}

\section{A R T I C L E I N F O}

\section{Keywords:}

Magnetic resonance spectroscopy

Bipolar disorder

Superior temporal gyrus

\begin{abstract}
A B S T R A C T
Background: Despite the diagnostic challenges in categorizing bipolar disorder subtypes, bipolar I and II disorders (BD-I and BD-II respectively) are valid indices for researchers. Subtle neurobiological differences may underlie clinical differences between mood disorder subtypes. The aims of this study were to investigate neurochemical differences between bipolar disorder subtypes.

Methods: Euthymic BD-II patients $(n=21)$ are compared with BD-I $(n=28)$ and healthy comparison subjects (HCs, $n=30$ ). Magnetic Resonance Imaging (MRI) and proton spectroscopy $\left({ }^{1} \mathrm{H}\right.$ MRS) were performed on a 3T Siemens Tim Trio system. MRS voxels were located in the left/right superior temporal cortices, and spectra acquired with the single voxel Point REsolved Spectroscopy Sequence (PRESS). The spectroscopic data were analyzed with LCModel (Version 6.3.0) software.

Results: There were significant differences between groups in terms of glutamate $[F=6.27, p=0.003]$, glutamate + glutamine $[F=6.08, p=0.004]$, inositol containing compounds (Ino) $(F=9.25, p<0.001)$, NAA $[F=7.63, p=0.001]$ and creatine + phosphocreatine $[F=11.06, p<0.001]$ in the left hemisphere and Ino $[F=5.65, p=0.005]$ in the right hemisphere. Post-hoc comparisons showed that the BD-I disorder group had significantly lower metabolite levels in comparison to the BD-II and the HC groups.

Limitations: This was a cross-sectional study with a small sample size. In addition, patients were on various psychotropic medications, which may have impacted the results.

Conclusions: Neurochemical levels, in the superior temporal cortices, measured with ${ }^{1} \mathrm{H}-\mathrm{MRS}$ discriminated between BD-II and BD-I. Although further studies are needed, one may speculate that the superior temporal cortices (particularly left hemispheric) play a critical role, whose pathology may be related to subtyping bipolar disorder.
\end{abstract}

\section{Introduction}

Despite the diagnostic challenges in categorizing bipolar disorder subtypes, bipolar I disorder (BD-I) and bipolar II disorder (BD-II) are valid categorical indices for researchers (Phillips and Kupfer, 2013; Parker and Fletcher, 2014). Along with depressive episodes, presence of manic or hypomanic episodes is the major difference between BD-I and
BD-II disorders. Despite the valid diagnostic boundaries, only limited studies have reported neurobiological differences between the subtypes (Phillips and Kupfer, 2013; Parker and Fletcher, 2014; McGrath et al., 2014).

Genetic studies have identified differences between BD-I and BD-II (Lee et al., 2011; Uemura et al., 2011). Psychosis (Parker and Fletcher, 2014), neurotoxic processes (Yumru et al., 2009; Uemura

\footnotetext{
* Corresponding author at: Department of Psychiatry, Ankara Yildirim Beyazit University Medical School, Universities Region, Ihsan Dogramaci Boulevard, No: 6, Bilkent Cankara, Ankara, Turkey.

E-mail address: miatagun@ybu.edu.tr (M.İ. Atagün).
} 
et al., 2011) and cognitive dysfunction (Bora et al., 2011) are more prevalent in BD-I compared to BD-II. Magnetic resonance imaging (MRI) studies have reported that BD-I had more severe findings in comparison to BD-II in volumes (Hauser et al., 2000), white matter lesions (Ambrosi et al., 2013) and functional connectivity (Li et al., 2012). Neurochemistry may also differ between the subtypes.

Proton magnetic resonance spectroscopy $\left({ }^{1} \mathrm{H}-\mathrm{MRS}\right)$ can quantify specific neurochemical compounds in the brain in vivo. It has been observed in ${ }^{1} \mathrm{H}$-MRS that mood state shifts and medications alter metabolites in BD-I (Moore et al., 2007). However, only a few MRS studies have compared BD-I and BD-II. No consistent finding has been reported between the subtypes in the frontal (Winsberg et al., 2000) or temporal (Hamakawa et al., 1998; Atagun et al., 2017) lobes, or the basal ganglia (Silverstone et al., 2004). Only one study detected increased choline concentrations in the basal ganglia in BD-II in comparison to BD-I (Kato et al., 1996).

Patients with bipolar disorder showed fronto-temporal dysconnectivity (Ozerdem et al., 2011) and cortical thinning was specific to superior temporal cortex in BD-I (Hanford et al., 2016). Furthermore, auditory processing deficits in bipolar I disorder are related to left hemisphere superior temporal cortices (McCarley et al., 2008). Moreover, it has been postulated that shared neurochemical abnormalities in superior temporal cortices by schizophrenia and bipolar disorder might be a common feature of psychosis (Nudmamud et al., 2003). On the other hand, superior temporal cortices may have unique local metabolic disturbances in both schizophrenia and bipolar disorder (McNamara et al., 2014). These findings have highlighted the pathology of superior temporal lobes in bipolar disorder. However, it is not clear whether there is difference between subtypes of bipolar disorder.

In this study, we aimed to compare neurochemical differences between BD-I and BD-II disorders utilizing ${ }^{1} \mathrm{H}$-MRS in superior temporal cortices. Our hypothesis was that there might be neurochemical differences between the subtypes of bipolar disorder. To our knowledge, this is the first ${ }^{1} \mathrm{H}-\mathrm{MRS}$ study to assess superior temporal lobes in BD-I and BD-II. In vivo assessment of metabolite levels in superior temporal lobes may show differences in pathogenesis of the subtypes of bipolar disorder.

\section{Methods}

All participants provided a written informed consent before study participation. This study was approved by the local Ethical Committee. In a recent article, we have reported abnormal metabolite levels in the superior temporal lobes of participants with BD-I (Atagün et al., 2015). We further enrolled patients with BD-II $(n=21$, mean age $=38.38 \pm 13.84,12$ women/9 men and compared with our BD-I sample and healthy comparison subjects (HCs). All patients were euthymic during the study participation. Clinical evaluation tools were Structured Clinical Interview according to the DSM-IV (SCID-I) (First et al., 1996), Young Mania Rating Scale (YMRS) (Young et al., 1978) and Hamilton Depression Rating Scale (HDRS) (Hamilton, 1960).

Data acquisition processes were performed with a $3.0 \mathrm{~T}$ Siemens MAGNETOM TIM Trio MR system (Siemens, Erlangen, Germany) and a 32 channel phased-array head coil. $\mathrm{T}_{1}$ anatomical MRI [MPRAGE sequence, parameters were TE: 3.02, TR: $2000 \mathrm{~ms}$, FOV phase: 100, FOV read: 215 , slice thickness: 0.84 , number of slices: 192$]$ and spectroscopy voxels [single voxel Point REsolved Spectroscopy Sequence (PRESS); $8 \mathrm{~cm}^{3}$ voxels $(2 \mathrm{~cm} \times 2 \mathrm{~cm} \times 2 \mathrm{~cm})$; TE: $30 \mathrm{~ms}$, TR: $2000 \mathrm{~ms}, 128$ averages] were recorded to evaluate left and right hemisphere superior temporal lobes which host Heschl's gyrus and planum temporale (Supplementary Fig. 1a). $B_{0}$ shimming was manually applied around the voxel. LCModel software (Version 6.3.0) was used to quantify the metabolite levels (Provencher, 2001), using water as internal reference. Representative spectra (Supplementary Fig. 1b) and quantified metabolites are presented in the supplement. Gray matter, white matter and cerebro-spinal fluid (CSF) contributions to the voxels were analyzed in T1 weighted structural MRI with Statistical Parametric Mapping-8 (SPM-8) software [Welcome Department of Imaging Neuroscience, London, UK; (http://www.fil.ion.ucl.ac.uk/spm/software/spm8/)] (for further details please see Atagün et al., 2015).

\subsection{Statistical analysis}

Statistical analyses were performed with SPSS 21 (IBM, Armonk, NY, USA) software. Distribution characteristics of the data are assessed with Kolmogorov-Smirnov and Shapiro-Wilks' Tests. Independent samples $t$ Test or Mann-Whitney $U$ Tests were performed for two independent group comparisons. Categorical variables were compared with chi-square test. Correlations were examined with Pearson's and Spearman's Correlation Tests. Univariate Analysis of Variance (ANOVA) and Analysis of Covariance (ANCOVA) was performed for three group comparisons for main effects. Chlorpromazine dose equivalents of antipsychotics, age, gender, age at onset, number of episodes, smoking status and alcohol consuming were available variables for covariate analysis. Interaction effects for grey matter, white matter and CSF contributions to the voxels were checked with interaction effect analysis in univariate ANOVA. Kruskal-Wallis Test was used for the comparison of non-Gaussian distributed variables. Post-hoc tests were Bonferroni or Tamhane Tests according to Levene's Homogeneity of Variance Test. Since 6 ANOVAs were performed for 6 metabolites (Glutamate, glutamate + glutamine, inositol containing compounds, creatine + phosphocreatine, choline containing compounds, $n$-Acetyl Aspartate) in the two hemispheres, a Bonferroni correction was applied to the $\mathrm{p}$ values $(6$ metabolites $\times 2$ hemispheres $=12 ; 0.05$ / $12=0.0041$ ) and level for statistical significance was 0.0041 for metabolite level comparisons. For all other comparisons, cut-off for statistical significance was 0.05 .

\section{Results}

Groups were similar in terms of age, gender, and education (Table 1). Smokers were more frequent in the patient groups $[F=6.99$, $p=0.030]$ and alcohol consumers were more frequent in the BD-II patient group $\left[\chi^{2}=9.69, p=0.008\right]$.

Comparison of the metabolite levels revealed that there were significant differences between groups in terms of glutamate $[F=6.27$, $p=0.003]$, glutamate + glutamine $[F=6.08, p=0.004]$, inositol containing compounds (Ino) $[F=9.25, p<0.001]$, NAA $[F=7.63$, $p=0.001]$ and creatine + phosphocreatine $[F=11.06, p<0.001]$ in the left hemisphere (Table 2). Post-hoc comparisons showed that the BD-I disorder group had significantly lower metabolite levels in comparison to the BD-II and the HC groups (Table 2). There was no significant difference between the groups in terms of voxel segmentation data (gray/white matter and cerebrospinal fluid content, results are presented in Table 2).

Correlation analysis was performed with Glu, Glx, NAA, Ino, Cr, age, age at onset, duration of the disease, number of episodes, serum lithium levels, serum valproate levels, chlorpromazine equivalent doses of antipsychotics, Young Mania Rating Scale and Hamilton Depression Rating Scale scores variables. There were significant correlations between right hemisphere N-Acetyl Aspartate (NAA) levels and age at disease onset $\left(r^{2}=-0.58, p=0.009\right)$, number of hypomanic episodes $\left(r^{2}=-0.51, p=0.032\right)$ in the BD-II group. ANCOVA showed that right hemisphere NAA levels were associated with the YMRS scores $[F=5.36, p=0.027]$, the number of life-time hypomanic episodes $[F=8.69, p=0.022]$, age of disease onset $[F=5.86, p=0.006]$ in the BD-II group. No other significant correlation was observed. Because we presented the BD-I group findings in the past, here we only present correlation analyses within the BD-II group. Medications are presented in Table 3 in the supplement. Metabolite levels did not differ between patients on/off lithium, valproate ( $p>0.05$ for all). There were no 
Table 1

Sociodemographic characteristics of the groups.

\begin{tabular}{|c|c|c|c|c|c|}
\hline & & BD-I $(n=28)$ & BD-II $(n=21)$ & HCs $(n=30)$ & $t / F / \chi^{2}$ \\
\hline \multicolumn{2}{|l|}{ Age } & $35.32 \pm 9.12$ & $38.38 \pm 13.84$ & $32.90 \pm 10.82$ & 1.47 \\
\hline \multicolumn{2}{|c|}{ Gender [women (\%)] } & $15(\% 53.6)$ & $12(\% 57.1)$ & $17(\% 56.7)$ & 0.15 \\
\hline \multicolumn{2}{|c|}{ Education* } & $10.89 \pm 4.86$ & $12.10 \pm 4.09$ & $9.97 \pm 3.16$ & 1.66 \\
\hline \multicolumn{2}{|c|}{ Age at disease onset* } & $23.57 \pm 8.66$ & $24.71 \pm 9.63$ & & -0.44 \\
\hline \multicolumn{2}{|c|}{ Duration of the disease $\mathrm{e}^{* *}$} & $92.21 \pm 107.69$ & $153.43 \pm 126.48$ & & -1.83 \\
\hline \multirow[t]{3}{*}{ Total number of } & Episodes & $7.82 \pm 5.46$ & $8.50 \pm 6.87$ & & -0.38 \\
\hline & Mania $¥$ & $2.77 \pm 2.18$ & $3.50 \pm 3.52$ & & -0.87 \\
\hline & Depression & $4.22 \pm 3.42$ & $4.90 \pm 4.15$ & & -0.61 \\
\hline \multicolumn{2}{|c|}{ Psychosis during episodes $n(\%)$} & $18(\% 64.3)$ & $8(\% 38.1) ¥$ & & 3.31 \\
\hline \multicolumn{2}{|c|}{ Nicotine $n(\%)$} & $18(\% 64.3)$ & $14(\% 66.7)$ & $10(\% 33.3)$ & 6.99 \\
\hline \multicolumn{2}{|l|}{ Alcohol $n(\%)$} & $2(\% 7.1)$ & $9(\% 40.9)$ & $5(\% 16.7)$ & 9.69 \\
\hline \multicolumn{2}{|l|}{ YMRS } & $1.07 \pm 1.61$ & $1.48 \pm 1.50$ & & -0.90 \\
\hline \multicolumn{2}{|l|}{ HAM-D } & $3.46 \pm 3.51$ & $3.38 \pm 3.20$ & & 0.09 \\
\hline
\end{tabular}

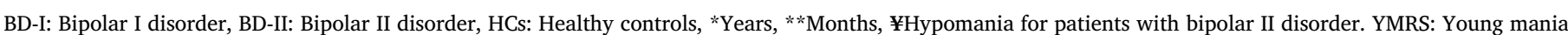

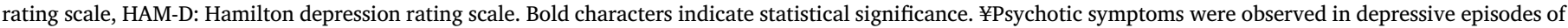
BD-II.

significant differences between psychotic $(n=26)$ and non-psychotic $(n=23)$ patients independent of diagnostic subtypes ( $p>0.05$ for all metabolites in both hemispheres). Psychosis, alcohol or nicotine consumption did not show any significant impact on the metabolite levels ( $p>0.05$; Supplementary Tables 4 and 5).

\section{Discussion}

In this study metabolite levels in the superior temporal cortices differed between the BD-I and BD-II groups. The BD-I group had significantly lower metabolite levels in comparison to the BD-II group. Further analysis showed that clinical factors such as psychosis, number of episodes and nicotine or alcohol consumption did not have any impact on metabolite levels. HDRS scores had inverse relationship between NAA and $\mathrm{Cr}$ in both right and left hemispheres and Ino and HDRS had correlation in the right hemisphere in BD-I (Atagun et al., 2015). Whereas NAA levels were negatively correlated with age at onset, number of hypomanic episodes and YMRS scores in the BD-II group.

Glutamatergic neurotransmission is maintained by glutamate to glutamine cycle and glial cells serve for the cycle. This cycle is differentially altered in manic and depressive states (Yüksel and Öngür, 2010). Globally Glx is elevated (Dager et al., 2004), however Glu levels are not significantly elevated in bipolar disorder (Yüksel and
Öngür, 2010; Atagun et al., 2015). Since Glx consist of glutamate plus glutamine, elevation of Glx is probably associated with increased glutamine levels. Quantification of glutamine is not possible with low magnetic field scanners, and thus there are not much studies in the literature. Glutamine levels in anterior cingulate cortex were found to be increased in bipolar disorder (Ongur et al., 2008). These findings may indicate that glutamate to glutamine cycle is disturbed in bipolar disorder. There may be a difference in glial physiopathology in BD-I and BD-II.

Inositol levels are found to be changed in bipolar disorder and mood stabilizers alter inositol levels by influencing phospho-inositol cycle (Silverstone and McGrath, 2009). However, the ${ }^{1} \mathrm{H}$-MRS literature regarding Ino levels is mixed (Silverstone and Mcgrath, 2009). Controlling for voxel locations, medications, field strength might be useful in future studies. NAA is a marker of mitochondrial function for oxidative metabolism and neuronal function/viability; decrease of NAA levels indicate neuronal dysfunction (Moffett et al., 2007). Cr metabolites are involved in energy metabolism by transporting high energy phosphorous groups; $\mathrm{Cr}$ compounds are highly diffusible and are considered to be stable throughout the brain. However, it has been determined that $\mathrm{Cr}$ is not stable in bipolar disorder and schizophrenia (Ongur et al., 2009). Thus, the use of $\mathrm{Cr}$ as an internal reference might be erroneous in bipolar disorder and schizophrenia. Correlation analysis revealed

Table 2

${ }^{1} \mathrm{H}-\mathrm{MRS}$ findings.

\begin{tabular}{|c|c|c|c|c|c|c|c|c|}
\hline & & BD-I $(n=28)$ & BD-II $(n=21)$ & HCs $(n=30)$ & $F$ & $p$ & Partial $\eta^{2}$ & Post-hoc \\
\hline \multirow[t]{9}{*}{ Right } & Glutamate & $1.31 \pm 0.16$ & $1.46 \pm 0.24$ & $1.43 \pm 0.18$ & 3.98 & 0.023 & 0.11 & \\
\hline & Glx & $1.87 \pm 0.26$ & $1.98 \pm 0.26$ & $1.95 \pm 0.26$ & 1.19 & 0.311 & 0.04 & \\
\hline & Inositol CC & $0.92 \pm 0.15$ & $1.02 \pm 0.21$ & $1.06 \pm 0.09$ & 5.65 & 0.005 & 0.25 & \\
\hline & Choline CC & $0.29 \pm 0.05$ & $0.30 \pm 0.05$ & $0.28 \pm 0.06$ & 1.04 & 0.358 & 0.03 & \\
\hline & NAA & $1.59 \pm 0.18$ & $1.72 \pm 0.18$ & $1.74 \pm 0.20$ & 4.94 & 0.010 & 0.13 & \\
\hline & $\mathrm{Cr}$ & $1.12 \pm 0.14$ & $1.27 \pm 0.19$ & $1.18 \pm 0.18$ & 4.38 & 0.016 & 0.11 & \\
\hline & Gray matter* & $56.83 \pm 4.78$ & $57.50 \pm 13.89$ & $58.00 \pm 4.87$ & 0.14 & 0.870 & $<0.01$ & \\
\hline & White matter* & $28.91 \pm 8.09$ & $29.01 \pm 5.11$ & $30.58 \pm 6.47$ & 0.51 & 0.604 & 0.01 & \\
\hline & $\mathrm{CSF}^{*}$ & $13.58 \pm 7.20$ & $13.00 \pm 11.72$ & $10.74 \pm 5.49$ & 0.93 & 0.401 & 0.02 & \\
\hline \multirow[t]{9}{*}{ Left } & Glutamate & $0.82 \pm 0.11$ & $0.99 \pm 0.25$ & $0.95 \pm 0.13$ & 6.27 & 0.003 & 0.15 & BD-I $<$ HCs,BD-II \\
\hline & Glx & $1.11 \pm 0.18$ & $1.36 \pm 0.37$ & $1.25 \pm 0.18$ & 6.08 & 0.004 & 0.15 & BD-I $<$ HCs,BD-II \\
\hline & Inositol CC & $0.57 \pm 0.12$ & $0.72 \pm 0.17$ & $0.67 \pm 0.09$ & 9.25 & $<0.001$ & 0.21 & BD-I $<$ HCs,BD-II \\
\hline & Choline CC & $0.17 \pm 0.03$ & $0.20 \pm 0.04$ & $0.19 \pm 0.03$ & 4.18 & 0.019 & 0.11 & \\
\hline & NAA & $1.02 \pm 0.13$ & $1.23 \pm 0.29$ & $1.17 \pm 0.13$ & 7.63 & 0.001 & 0.18 & BD-I $<$ HCs,BD-II \\
\hline & $\mathrm{Cr}$ & $0.71 \pm 0.11$ & $0.90 \pm 0.19$ & $0.81 \pm 0.10$ & 11.06 & $<0.001$ & 0.23 & BD-I $<$ HCs,BD-II \\
\hline & Gray matter* & $57.03 \pm 4.42$ & $57.05 \pm 14.84$ & $59.78 \pm 5.73$ & 0.85 & 0.432 & 0.22 & \\
\hline & White matter* & $31.06 \pm 9.46$ & $30.67 \pm 7.77$ & $30.51 \pm 6.72$ & 0.04 & 0.966 & $<0.01$ & \\
\hline & $\mathrm{CSF}^{*}$ & $10.25 \pm 7.48$ & $11.85 \pm 11.27$ & $9.38 \pm 4.56$ & 0.59 & 0.556 & 0.02 & \\
\hline
\end{tabular}

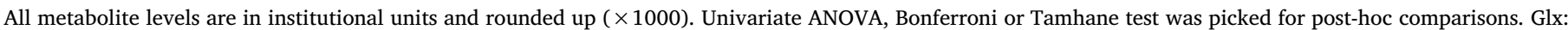

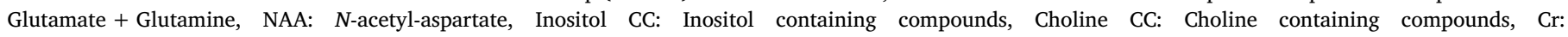
Creatine + Phosphocreatine, CSF: Cerebrospinal fluid content. *Voxel segmentation data (percentage), bold indicate statistical significance. 
differences between BD-I and BD-II. NAA levels are negatively correlated with number of hypomanic episodes and age at disease onset in BD-II. Whereas in BD-I HDRS scores were negatively correlated with Ino and NAA (reported in Atagun et al., 2015).

Small sample size and being a cross-sectional study are major limitations of this study. Furthermore, patients were on various psychotropic medications and medications are known to alter the metabolites measured by ${ }^{1} \mathrm{H}$-MRS (Moore et al., 2000; Moore et al., 2007). Because of its anatomical adjacency to cranium, $B_{0}$ and $B_{1}$ inhomogeneity are high in temporal lobe (Juchem and de Graff, 2016). $\mathrm{B}_{0}$ shimming was performed, however $B_{1}$ shimming was not available in this study.

To conclude, levels of metabolites are decreased in BD-I in comparison to BD-II and healthy controls in this study. These findings may indicate that neuronal dysfunction and metabolic decline in the left (dominant) hemisphere superior temporal lobe is specific to BD-I. Although further studies are needed, it can be postulated that pathology of the left hemispheric superior temporal lobe might be more severe in BD-I in comparison to BD-II. Our findings indicate that the left hemispheric superior temporal lobe metabolite levels in BD-I are declined similar to psychotic spectrum disorders rather than BD-II. Further studies comparing manic and hypomanic episodes of BD-I and BD-II may reveal metabolic differences between the subtypes.

\section{Author statement}

\section{Contributors}

MIA: Prepared the initial study protocol and procedures, data collection, interpretation of the results, wrote the manuscript.

EMS: Data analysis, interpretation of the results and preparation of the manuscript.

SSC: Initial study procedures and data collection.

GKU: Initial study procedures and data collection.

SUK: Initial study procedures and data collection.

AC: Reviewed the protocol, initial study procedures and data collection.

OA: Initial study procedures, data collection and data analysis.

MLP: Scientific supervision during the preparation of the protocol, interpretation of the results and supervision for the manuscript.

CMM: Scientific supervision during the preparation of the protocol, data analysis, interpretation of the results and supervision for the manuscript.

DO: Scientific supervision during the preparation of the protocol, data analysis, interpretation of the results and supervision for the manuscript.

\section{Role of funding source}

The study was funded by Scientific Research Projects Committee of the Ankara Yıldırım Beyazıt University (Project Code: 803) and NIMH grant to CMM (MH073998) and NIH grant to DO K24 (MH104449). Dr. Phillips acknowledged the support of the Pittsburgh Foundation.

\section{Conflict of interest}

Dr. Kaymak participated in Otsuka's clinical trial (ClinicalTrials.gov Identifier: NCT01129882.), Dr. Can participated in Janssen Cilag's clinical trial (ClinicalTrials.gov Identifier: NCT02782104). Other authors declare no conflict of interest.

\section{Acknowledgment}

The authors would like to thank to Professor Ergin Atalar and UMRAM staff. The authors also appreciate technical help by Dinesh Deelchand, Dr. Uzay Emir and Gülin Öz (from the Center for Magnetic Resonance Research, Minneapolis, MN, USA) during voxel segmentation

\section{Supplementary materials}

Supplementary material associated with this article can be found, in the online version, at doi:10.1016/j.jad.2018.04.010.

\section{References}

Ambrosi, E., Rossi-Espagnet, M.C., Kotzalidis, G.D., Comparelli, A., Del Casale, A., Carducci, F., Romano, A., Manfredi, G., Tatarelli, R., Bozzao, A., Girardi, P., 2013. Structural brain alterations in bipolar disorder II: a combined voxel-based morphometry (VBM) and diffusion tensor imaging (DTI) study. J. Affect. Disord. 150, 610-615.

Atagün, M.İ., Şıkoğlu, E.M., Can, S.S., Karakaş-Uğurlu, G., Ulusoy-Kaymak, S., Çayköylü, A., Algın, O., Phillips, M.L., Moore, C.M., Öngür, D., 2015. Investigation of Heschl's gyrus and planum temporale in patients with schizophrenia and bipolar disorder: A proton magnetic resonance spectroscopy study. Schizophr. Res. 161, 202-209.

Atagün, M.İ., Şıkoğlu, E.M., Soykan, Ç., Can, S.S., Ulusoy-Kaymak, S., Çayköylü, A., Algın, O., Phillips, M.L., Öngür, D., Moore, C.M., 2017. Perisylvian GABA levels in schizophrenia and bipolar disorder. Neurosci. Lett. 637, 70-74.

Bora, E., Yücel, M., Pantelis, C., Berk, M., 2011. Meta-analytic review of neurocognition in bipolar II disorder. Acta Psychiatr. Scand. 123, 165-174.

Dager, S.R., Friedman, S.D., Parow, A., Demopulos, C., Stoll, A.L., Lyoo, I.K., Dunner, D.L., Renshaw, P.F., 2004. Brain metabolic alterations in medication-free patients with bipolar disorder. Arch. Gen. Psychiatry 61, 450-458.

First, M.D., Gibbon, M., Spitzer, R.L., Gibbon, M., Williams, J.B.W., 1996. User's Guide for the Structured Interview for DSM-IV Axis I Disorders Research Version (SCID-I, Version 2.0). Biometrics Research, New York.

Hamakawa, H., Kato, T., Murashita, J., Kato, N., 1998. Quantitative proton magnetic resonance spectroscopy of the basal ganglia in patients with affective disorders. Eur. Arch. Psychiatr. Clin. Neurosci. 248, 53-58.

Hamilton, M., 1960. A rating scale for depression. J. Neurol. Neurosurg. Psychiatry 23, 56-62.

Hanford, L.C., Nazarov, A., Hall, G.B., Sassi, R.B., 2016. Cortical thickness in bipolar disorder: a systematic review. Bipolar Disord 18, 4-18.

Hauser, P., Matochik, J., Altshuler, L.L., Denicoff, K.D., Conrad, A., Li, X., Post, R.M., 2000. MRI-based measurements of temporal lobe and ventricular structures in patients with bipolar I and bipolar II disorders. J. Affect. Disord. 60, 25-32.

Juchem, C., de Graaf, R.A., 2016. B0 magnetic field homogeneity and shimming for in vivo magnetic resonance spectroscopy. Anal Biochem 529, 17-29.

Kato, T., Hamakawa, H., Shioiri, T., Murashita, J., Takahashi, Y., Takahashi, S., Inubushi, T., 1996. Choline-containing compounds detected by proton magnetic resonance spectroscopy in the basal ganglia in bipolar disorder. J. Psychiatry Neurosci. 21, 248-254.

Lee, S.Y., Chen, S.L., Chen, S.H., Huang, S.Y., Tzeng, N.S., Chang, Y.H., Wang, C.L., Lee, I.H., Yeh, T.L., Yang, Y.K., Lu, R.B., 2011. The COMT and DRD3 genes interacted in bipolar I but not bipolar II disorder. World J. Biol. Psychiatry 12, 385-391.

Li, C.T., Hsieh, J.C., Wang, S.J., Yang, B.H., Bai, Y.M., Lin, W.C., Lan, C.C., Su, T.P., 2012. Differential relations between frontolimbic metabolism and executive function in patients with remitted bipolar I and bipolar II disorder. Bipolar Disord 14, 831-842.

McCarley, R.W., Nakamura, M., Shenton, M.E., Salisbury, D.F., 2008. Combining ERP and structural MRI information in first episode schizophrenia and bipolar disorder. Clin. EEG Neurosci. 39 (2), 57-60.

McGrath, B.M., Wessels, P.H., Bell, E.C., Ulrich, M., Silverstone, P.H., 2014. Neurobiological findings in bipolar II disorder compared with findings in bipolar I disorder. Can. J. Psychiatry 49, 794-801.

McNamara, R.K., Rider, T., Jandacek, R., Tso, P., 2014. Abnormal fatty acid pattern in the superior temporal gyrus distinguishes bipolar disorder from major depression and schizophrenia and resembles multiple sclerosis. Psychiatry Res 215 (3), 560-567.

Moffett, J.R., Ross, B., Arun, P., Madhavarao, C.N., Namboodiri, A.M., 2007. NAcetylaspartate in the CNS: from neurodiagnostics to neurobiology. Prog. Neurobiol. 81, 89-131.

Moore, C.M., Biederman, J., Wozniak, J., Mick, E., Aleardi, M., Wardrop, M, Dougherty, M., Harpold, T., Hammerness, P., Randall, E., Lyoo, I.K., Renshaw, P.F., 2007. Mania, glutamate/glutamine and risperidone in pediatric bipolar disorder: a proton magnetic resonance spectroscopy study of the anterior cingulate cortex. J. Affect. Disord. 99, 19-25.

Moore, G.J., Bebchuk, J.M., Hasanat, K., Chen, G., Seraji-Bozorgzad, N., Wilds, I.B., Faulk, M.W., Koch, S., Glitz, D.A., Jolkovsky, L., Manji, H.K., 2000. Lithium increases Nacetyl-aspartate in the human brain: in vivo evidence in support of bcl-2's neurotrophic effects? Biol. Psychiatry 48, 1-8.

Nudmamud, S., Reynolds, L.M., Reynolds, G.P., 2003. N-acetylaspartate and NAcetylaspartylglutamate deficits in superior temporal cortex in schizophrenia and bipolar disorder: a postmortem study. Biol. Psychiatry 53, 1138-1141.

Ongur, D., Jensen, J.E., Prescot, A.P., Stork, C., Lundy, M., Cohen, B.M., Renshaw, P.F., 2008. Abnormal glutamatergic neurotransmission and neuronal-glial interactions in acute mania. Biol. Psychiatry 64 (8), 718-726.

Ongur, D., Prescot, A.P., Jensen, J.E., Cohen, B.M., Renshaw, P.F., 2009. Creatine abnormalities in schizophrenia and bipolar disorder. Psychiatry Res 172 (1), 44-48.

Özerdem, A., Güntekin, B., Atagün, I., Turp, B., Başar, E., 2011. Reduced long distance gamma $(28-48 \mathrm{~Hz})$ coherence in euthymic patients with bipolar disorder. J. Affect. Disord. 132 (3), 325-332. 
Parker, G., Fletcher, K., 2014. Differentiating bipolar I and II disorders and the likely contribution of DSM-5 classification to their cleavage. J. Affect. Disord. 152-154, $57-64$.

Phillips, M.L., Kupfer, D.J., 2013. Bipolar disorder diagnosis: challenges and future directions. Lancet 381, 1663-1671.

Provencher, S.W., 2001. Automatic quantitation of localized in vivo $1 \mathrm{H}$ spectra with LCModel. NMR Biomed 14, 260-264.

Silverstone, P.H., Asghar, S.J., O'Donnell, T., Ulrich, M., Hanstock, C.C., 2004. Lithium and valproate protect against dextro-amphetamine induced brain choline concentration changes in bipolar disorder patients. World J. Biol. Psychiatry 5, 38-44.

Silverstone, P.H., McGrath, B.M., 2009. Lithium and valproate and their possible effects on themyo-inositol second messenger system in healthy volunteers and bipolar patients. Int. Rev. Psychiatry 21 (4), 414-423.
Uemura, T., Green, M., Corson, T.W., Perova, T., Li, P.P., Warsh, J.J., 2011. Bcl-2 SNP rs956572 associates with disrupted intracellular calcium homeostasis in bipolar I disorder. Bipolar Disord 13, 41-51.

Winsberg, M.E., Sachs, N., Tate, D.L., Adalsteinsson, E., Spielman, D., Ketter, T.A., 2000 Decreased dorsolateral prefrontal $\mathrm{N}$-acetylaspartate in bipolar disorder. Biol. Psychiatry 47, 475-481.

Young, R.C., Biggs, J.T., Ziegler, V.E., Meyer, D.A., 1978. A rating scale for mania: reliability, validity and sensitivity. Br. J. Psychiatry 133, 429-435.

Yüksel, C., Öngür, D., 2010. Magnetic resonance spectroscopy studies of glutamate-related abnormalities in mood disorders. Biol. Psychiatry 68 (9), 785-794.

Yumru, M., Savas, H.A., Kalenderoglu, A., Bulut, M., Celik, H., Erel, O., 2009. Oxidative imbalance in bipolar disorder subtypes: A comparative study. Prog.

Neuropsychopharmacol. Biol. Psychiatry 33, 1070-1074. 